\title{
ANALYSIS OF PROANTHOCYANIDINS IN WILD-TYPE AND MUTANT BARLEY (HORDEUM VULGARE L.)
}

\author{
by
}

\author{
BARBRO JENDE-STRID and BIRGER LINDBERG MØLLER
}

Department of Physiology, Carlsberg Laboratory, Gamle Carlsberg Vej 10, DK-2500 Copenhagen Valby

\begin{abstract}
Keywords: Beer stability, proanthocyanidin-free barley, high performance liquid chromatography, flavonoids
\end{abstract}

The proanthocyanidin composition of wild-type barley was analyzed by high-performance liquid chromatography. The use of authentic standards allowed tentative identification of procyanidin B-3, prodelphinidin B-3, and catechin as the major constituents. The proanthocyanidin content of different barley varieties (wild types) was found to be qualitatively and quantitatively similar. Mutants belonging to different gene loci and deficient in the biosynthesis of proanthocyanidins and other flavonoids were characterized.

\section{INTRODUCTION}

Permanent haze and chill haze in beer are caused by the precipitation of protein with polyphenols derived from barley and hops, the starting materials in the beer production (16, 20). Hitherto, haze formation has been prevented by filtration of the beer through polyvinylpolypyrrolidone or silica gel (19). Alternatively the beer proteins are degraded with proteolytic enzymes or various additives such as antioxidants are used to prevent precipitation. As the most important phenols in haze formation are the proanthocyanidins (5), chemical stabilization can be made superfluous by the use of barley varieties in which the biosynthesis of these constituents is genetically blocked. Towards this goal 173 induced proanthocyanidin deficient barley mutants have been isolated and eleven of these used for experimental malting and brewing. It has been shown that stable beer of excellent taste can be obtained from such a variety of barley $(29,30)$. It is not known whether specific or all proanthocyanidins of barley are equally efficient in haze formation. Quantitative as well as qualitative analyses of these constituents in the mutants and their wild-

Abbreviations: $\mathrm{cat}=$ catechin; gal $=$ gallocatechin $; \mathrm{HPLC}=$ high performance liquid chromatography $; \mathrm{PC}=$ procyanidin; $P D=$ prodelphinidin. 
types are essential to answer this question and will also provide basic information on the biosynthesis of proanthocyanidins. The quantitative and qualitative analysis of polyphenols is difficult because of the large number of different components normally present including products of various degrees of polymerization which are either natural constituents or are formed during extraction procedures. Traditional quantitative analysis of proanthocyanidins are based on colorimetric measurements after their conversion to anthocyanidins upon heating in acid solution $(9,18)$. This conversion is not quantitative and does not permit distinction among different types of proanthocyanidins.

In recent years gas chromatography and high performance liquid chromatography (HPLC) have been used for separation and determination of flavonoids. VANCRAENENBROECK et al. (27) studied the influence of proanthocyanidin and catechin content in beer on colloidal stability with the aid of gas chromatography and a method for simultaneous quantitative determination of biflavans and catechins was devised. However, proanthocyanidins of a higher molecular weight are less volatile and therefore difficult to analyse.

High performance liquid chromatography has proven to be an excellent tool for the separation of complex mixtures of flavonoids from plant material $(8,24,25,31)$. The HPLC method has also been used to analyse the proanthocyanidin content of cider (17). Of the different types of column packings used (adsorption, gel permeation, reversed phase and partition) a system based on reversed phase chromatography seemed most promising. Recently, the separation obtained using HPLC on reversed phase columns has been greatly improved by Jerumanis (14), who also used this method to analyse proanthocyanidins and catechins from malt, hops and beer. In the present study we have investigated the proanthocyanidin and catechin composition of the grain from barley varieties and single gene mutants with an altered flavonoid content.

\section{MATERIALS AND METHODS}

\subsection{Plant material}

Grains of the malting barley variety Nordal (Hordeum vulgare L.) were used as standard material to develop extraction methods and procedures. In addition to Nordal, the two barley varieties Gula and Triumph were analysed for proanthocyanidins. The anthocyanin, proanthocyanidin and catechin deficient mutants used in this study were obtained from our collection of 230 induced mutants. The mutants ant 2-15 and ant 13-13 were obtained from the variety Foma, mutants ant 2-20, ant 17-139 and ant 18-141 were induced in Nordal, the mutants ant 13152, ant 17-148 and ant 17-150 are derived from Triumph, while the mutants ant 18-159, ant 18-168 and ant 19-109 originate from Gula, Ark-Royal and Alf, respectively. The five genes ant 2, ant 13, ant 17, ant 18 and ant 19 have been identified by diallelic crosses. Among 38 different genetically analysed mutants, eight mutations were found in the gene ant 13, fourteen in the gene ant 17, fifteen in the gene ant 18 , and one in the gene ant 19. All mutants used except for ant 19-109 and ant 2-15 are deficient in anthocyanins, catechins and proanthocyanidins. The mutant ant 19-109 has a normal anthocyanin content in its green parts but is proanthocyanidin and catechin free in the grain, whereas ant 2-15 is anthocyanin free but produces a normal amount of proanthocyanidin and catechin.

\subsection{Extraction procedures}

Barley grains $(10 \mathrm{~g})$ were ground into a fine powder using a Brown Type MX 32 homogenizer. The freshly ground flour was extracted with $50 \mathrm{ml}$ of $75 \%$ acetone (15) under vigorous shaking. The slurry was centrifuged for $5 \mathrm{~min}$ at $25.000 \times \mathrm{g}$ after which the pellet obtained was re-extracted twice. The combined supernatants thus obtained were, depending on their future use, processed by either procedure A or B as described below. Procedure A: The acetone extract (approx. $150 \mathrm{ml}$ ) was delipidated with $3 \times 50 \mathrm{ml}$ of petroleum benzine (bp. $40-60^{\circ}$ ). The aqueous acetone phase was evaporated at $20^{\circ} \mathrm{C}$ on a Rotary evaporator to a final volume of approx. $30 \mathrm{ml}$. At this stage most of the acetone is removed and the extract becomes cloudy. To prevent precipitation upon standing, $5 \mathrm{ml}$ of $96 \%$ ethanol was immediately added. The sample was clarified by centrifugation $(9.000 \times \mathrm{g}, 15 \mathrm{~min})$ before HPLC analysis. 
Procedure B: The acetone extract (approx. $150 \mathrm{ml}$ ) was delipidated in a separatory funnel by use of $50 \mathrm{ml}$ of benzene. The aqueous bottom phase was collected and evaporated to a small volume to remove remaining acetone and benzene and was then extracted with $4 \times 15 \mathrm{ml}$ of ethylacetate (14). The combined ethyl acetate phases were evaporated to dryness $\left(40^{\circ}\right)$ in a Rotary evaporator and the residue dissolved in $0.5 \mathrm{ml}$ of $96 \%$ ethanol. The extract was clarified by centrifugation as described above.

\subsection{High performance liquid chromatography}

Analyses were performed using a Waters Assoc. (Milford, Mass., US) instrument composed of a Model 6000 A solvent delivery system, a Model U6K injector, a Model 450 variable wavelength detector, a Data Module and a System Controller. All separations were carried out on a $\mu$ Bondapak Phenyl column (Waters Assoc.) by use of a gradient system. The initial conditions of the gradient system was $2 \%$ acetic acid and this was changed to a final concentration of $10 \%$ acetic acid using program 8 of the System Controller and maintained at $10 \%$ acetic acid for sufficient time to elute all proanthocyanidins. The flow rate was 0.5 or $1.0 \mathrm{ml} \cdot \mathrm{min}^{-1}$ and the elution was monitored spectrophotometrically at $281 \mathrm{~nm}$.

\subsection{Assays}

The major components absorbing at $281 \mathrm{~nm}$ were collected when eluted from the $\mu$ Bondapak Phenyl column. Each isolate was divided into aliquots, one of which was tested with vanillin (30) and the other hydrolyzed with $5 \mathrm{~N} \mathrm{HCl} /$ ethanol for $20 \mathrm{~min}$ at $80^{\circ}$ to localize proanthocyanidins and catechins. The absorption spectra of the latter fractions were measured to distinguish between cyanidin $(\lambda \max 535 \mathrm{~nm})$ and delphini$\operatorname{din}(\lambda \max 543 \mathrm{~nm})$ residues.

\subsection{Proanthocyanidin standards}

Samples of proanthocyanidins B-1, B-2, B-3, and B-4 were obtained from Dr. E. Haslam, Department of Chemistry, University of Sheffield, England and from Dr. L. Porter, Chemistry Division, D.S.I.R., Petone, New Zealand.
Standard mixtures of dimeric and trimeric proanthocyanidins isolated from barley were provided by cand. polyt. H. OutrRup, Department of Brewing Chemistry, Carlsberg Research Laboratory.

\section{RESULTS}

Analyses based on the vanillin test had shown that the proanthocyanidin content of the malting barley variety Nordal is similar to that of other barley varieties. This variety was used as a standard to develop an extraction procedure for the proanthocyanidins of barley. The procedure is based on repeated extraction of these components using $75 \%$ acetone followed by extensive removal of lipids through repetitive washings with petroleum benzine. HPLC analysis of the composition of the final extracts thus obtained is shown in Figure 1. The efficiency of each extraction step was evaluated by HPLC analysis. The first two acetone extracts were found to contain 82 and $17 \%$ of the total acetone extractable proanthocyanidin. No qualitative differences between the two extracts were observed. When the combined acetone extracts were washed with petroleum benzine no loss of proanthocyanidins could be detected.

A different method for the extraction of proanthocyanidins from malt, hops, and beer has been used by Jerumanis (14). This procedure was also tested except that the fractionation step involving polyamide chromatography was omitted. The proanthocyanidin pattern thus obtained is shown in Figure 2. It is both quantitatively and qualitatively different from that obtained with the previous extraction procedure. The total yield of proanthocyanidins is less than $50 \%$ and it is apparent that there is a specific loss of the more polar proanthocyanidins which cannot be quantitatively extracted from an aqueous phase into ethyl acetate. However, several peaks observed with method A are not proanthocyanidins and the number and size of these peaks are greatly reduced after ethylacetate extraction.

In order to decide which of the major peaks observed in the HPLC profiles were proanthocyanidins and catechins, a semi-preparative separation was carried out. Individual fractions were tested by the vanillin test and analysed spectrophotometrically for the presence of anthocyani- 
B. Jende-Strid \& B. L. Møller: Proanthocyanidins in barley

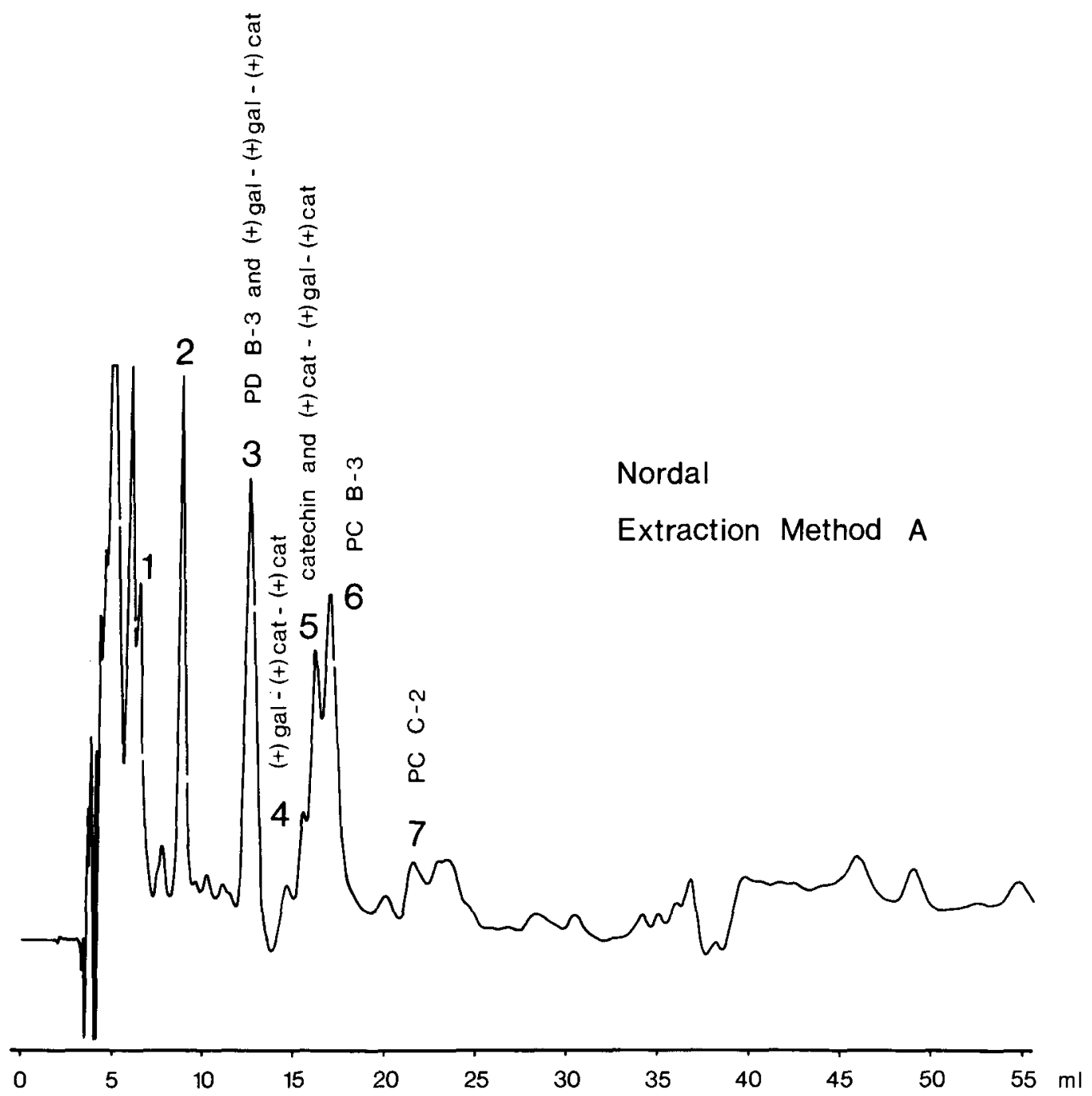

Figure 1. HPLC chromatogram of the acetone extractable proanthocyanidins in barley (Hordeum vulgare cv. Nordal).

dins after acid hydrolysis (Figure 3). Absorbance spectroscopy of the hydrolysates showed that the vanillin positive peaks can contain both catechin and gallocatechin units. The fractions giving the first vanillin positive peak (Fig. 3, *) did not yield anthocyanidins upon acid hydrolysis and do therefore not contain proanthocyanidins. Tentative assignment of some of the proanthocyanidin and catechin containing peaks was carried out with the help of standards as indicated in Figure 1 and Table I. Based on superimposable retention times of the known standards and observed peaks in the chromatograms, the major components were found to be prodelphinidin B-3, procyanidin B-3 and catechin. Proanthocyanidin containing peaks corresponding to the trimers $(+)$-gal-(+)-gal-(+)-cat (trimer-1), (+)-gal- $(+)-$ cat- $(+)$-cat (trimer-2), (+)-cat- $(+)$-gal- $(+)$-cat (trimer-3), and (+)-cat-(+)-cat-(+)-cat (procyanidin $\mathrm{C}-2$ = trimer -4 ) isolated from barley $(21,22)$ were also found in the wild-type chromatograms. However, these trimers are not well separated from the other major proanthocyanidin components and are therefore difficult to 


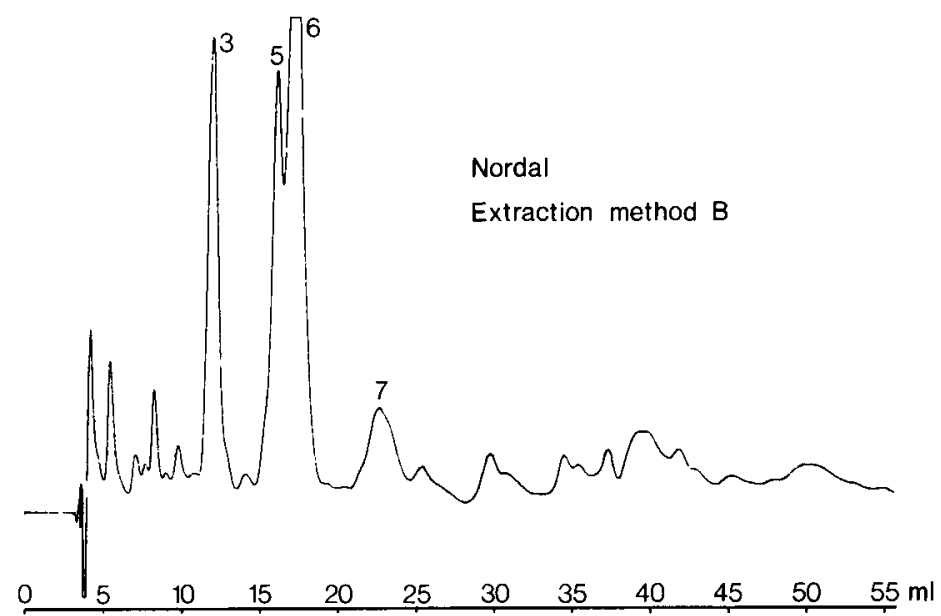

Figure 2. HPLC chromatogram of the ethyl acetate extractable proanthocyanidins of barley cv. Nordal. The amount injected was increased by a factor of six compared to the chromatograms in Figure 1.

quantitate with the exception of the trimer $(+)$ gal- $(+)$ cat- $(+)$ cat, which definitively is a minor component. The absorbance maxima for each of the assigned peaks were in all cases in agreement

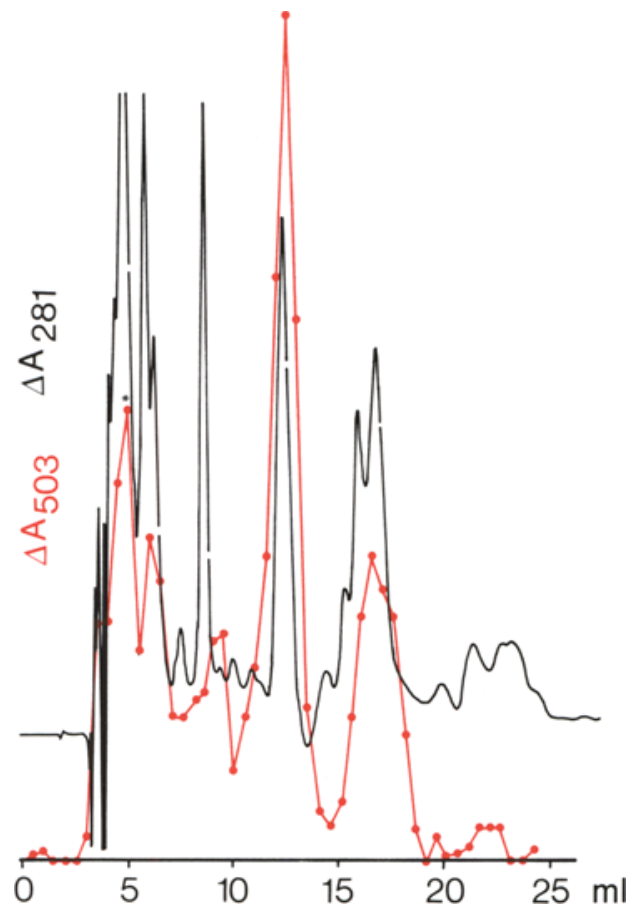

Figure 3. HPLC chromatogram of acetone extractable proanthocyanidins from Nordal barley contrasted with position of vanillin positive components. with the known composition of the standard. In addition to the proanthocyanidin peaks corresponding to known standards, two unknown proanthocyanidins are present. These were collected when eluted from the HPLC and were found upon acid hydrolysis and spectroscopy to contain both catechin and gallocatechin units.

The proanthocyanidin-deficient mutants studied were induced in different mother varieties. It was therefore of interest to examine whether the proanthocyanidin composition and content varied among these different varieties. The proanthocyanidin profiles of three such wildtypes were investigated and found to be similar although the ratios of prodelphinidins to procyanidins varied among the lines (Figures 1, 4 and 5). In order to quantify the amounts of proanthocyanidins and catechins present, known amounts of standards were injected into the HPLC. The corresponding peak areas were measured and used to calculate the amounts of proanthocyanidins and catechins present in the different varieties. Nordal was found to contain $130 \mathrm{mg}$ proanthocyanidin/100 g dry weight whereas the other two varieties Gula and Triumph contained 160 and $145 \mathrm{mg}$ proanthocyanidin/ $100 \mathrm{~g}$ dry weight, respectively.

The criteria used to identify proanthocyanidin-free mutants have been a negative vanillin test (30). This test is rather unspecific and quantitative results are difficult to obtain. It was therefore decided to test mutants in different 
Table 1

HPLC retention times of proanthocyanidin and catechin standards. Also listed are the retention times for major components in barley grain extracts.

\begin{tabular}{llcc}
\hline Peak & Compound & $\begin{array}{c}\text { Retention } \\
\text { time, mins. }\end{array}$ & $\begin{array}{c}\lambda \text {-max. nm after } \\
\text { hydrolysis }\end{array}$ \\
\hline 1. & Unknown, composed of procyanidin & & \\
2. & and prodelphinidin units & 12.65 & 538 \\
& Unknown, composed of procyanidin & 17.30 & 538 \\
and prodelphinidin units & 19.10 & 543 \\
3. & Trimer-1 and prodelphinidin B-3 & 24.75 & \\
& Trimer-1 [(+)-gal-(+)-gal-(+)-cat], standard & 24.90 & \\
& Prodelphinidin B-3, standard & 25.20 & \\
4. & Trimer-2 [(+)-gal-(+)-cat-(+)-cat], standard & 30.50 & \\
& Trimer-2 & 30.65 & \\
(+)-catechin, standard & 31.60 & 535 \\
6. & Trimer-3 [(+)-cat-(+)-gal-(+)-cat], standard & 31.80 & \\
& (+)-catechin and Trimer-3 & 31.90 & \\
& Procyanidin B-3 & 33.45 & \\
& Procyanidin B-3, standard & 33.50 & \\
& Procyanidin B-1, standard & 40.20 & \\
7. & Procyanidin C-2, standard & 42.10 & \\
& Procyanidin C-2 & 42.60 & \\
& (-)-epicatechin, standard & 47.70 & \\
& Procyanidin B-4, standard & 49.70 & 535 \\
& Procyanidin B-2, standard & 60.10 & \\
& Cyanidin, standard & & 543 \\
\hline
\end{tabular}

genes using the HPLC procedure. The analyses were carried out with both extraction procedures. HPLC analysis of mutants in the gene ant-13 (ant 13-13 and ant 13-152) showed the absence of proanthocyanidins. No peaks were found when the ethyl acetate procedure was followed (Figure 6), and the profile obtained was identical to that observed upon injection of solvent only (Figure 7). A major peak was observed in the Method A extract (Figure 8). This peak had an identic retention time to the proanthocyanidin containing peak 2 in the wildtype but gave a negative vanillin test and is therefore considered not to be a proanthocyanidin. Mutants tested in the gene loci ant 17 (Figure 9), ant 18 (Figure 10) and ant 19 (Figure 11) revealed a complex pattern of unidentified peaks of low intensity, some of which could represent proanthocyanidin precursors. Mutants in the locus ant 18 may contain small amounts of prodelphinidin and procyanidin B-3. The mutant ant 2-15, which is blocked in anthocyanin biosynthesis (13) had a proanthocyanidin pattern similar to that of the wild-type.

\section{DISCUSSION}

Proanthocyanidins are widely distributed in the plant kingdom $(1,4,15,26)$. Until recently, no good methods have been available for the isolation and characterization of proanthocyanidin polymers and most work has therefore been concentrated on the relatively simpler proanthocyanidin dimers and trimers $(26,28)$. Both the simple and polymeric proanthocyanidins are typically composed of procyanidin units and less commonly of prodelphinidin units (4). Other units such as propelargonidin and the flavan-3ols (+)-afzelechin and (-)epiafzelechin are rare (3).

The functions of proanthocyanidins in plants remain obscure. A known property of many 

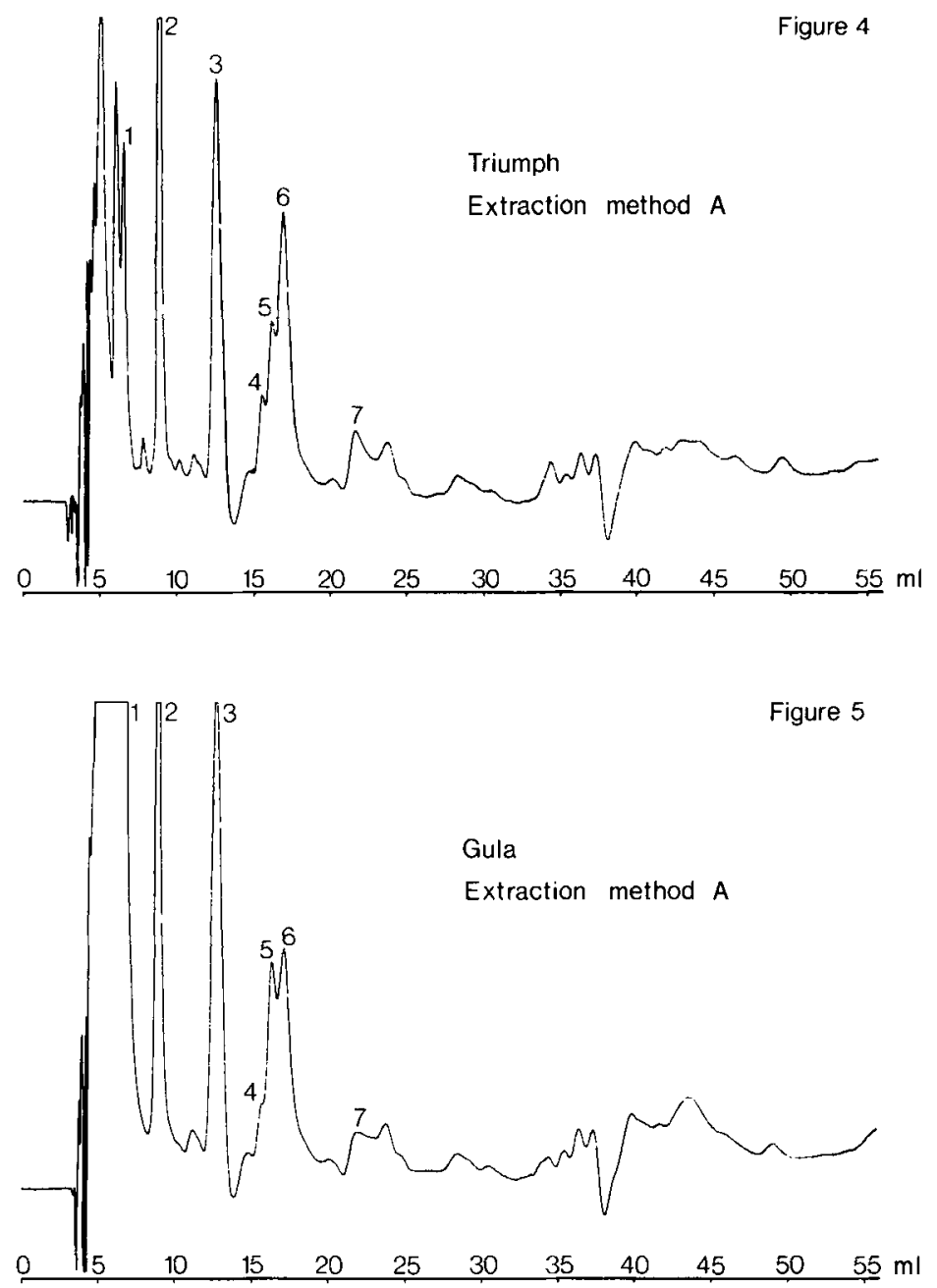

Figures $4 \& 5$. HPLC chromatogram of the acetone extractable proanthocyanidins from the barley varieties Triumph and Gula.

proanthocyanidins is their ability to associate with proteins forming insoluble complexes. Proanthocyanidins with higher molecular weights containing high levels of prodelphinidins $(10,15)$ seem to be the most effective in this respect. In the species examined, it has been found that this polymeric proanthocyanidin fraction constitutes between 90 and $99 \%$ of the total flavonoid content, the rest being composed of catechins and their dimers (15).

Sorghum and barley are the only cereals which contain proanthocyanidins. The Sorghum proanthocyanidins are located in the pericarp and have been identified as catechin, proanthocyanidin B-1 and a polymeric proanthocyanidin fraction with a molecular weight in the range of 1700-2000 (7). Sorghum varieties with a high proanthocyanidin content have a lower nutritional value compared to those with a low content (23).

The proanthocyanidins of barley are located in the aleuron layer of the grain. The pattern is complex and both procyanidins and prodelphinidins occur. JeRUManis (14) has reported procyanidin B-3, B-6 and C-2, an undefined prodelphinidin, catechin, and epicatechin as constituents of 
B. Jende-Strid \& B. L. Møller: Proanthocyanidins in barley

Figure 6

ant 13-13

Extraction method $B$

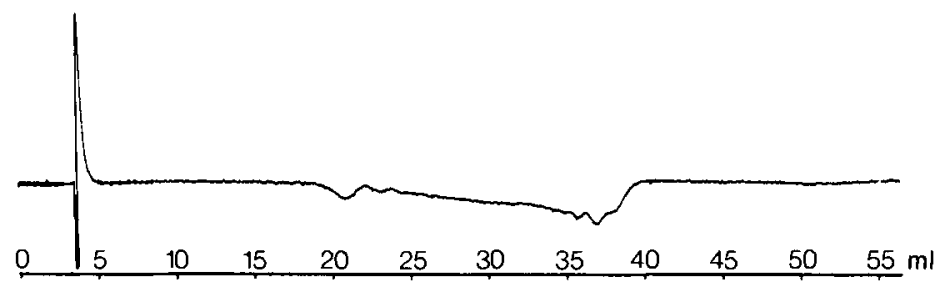

Figure 7

Background

$15 \mu \mathrm{l} 96 \%$ ethanol
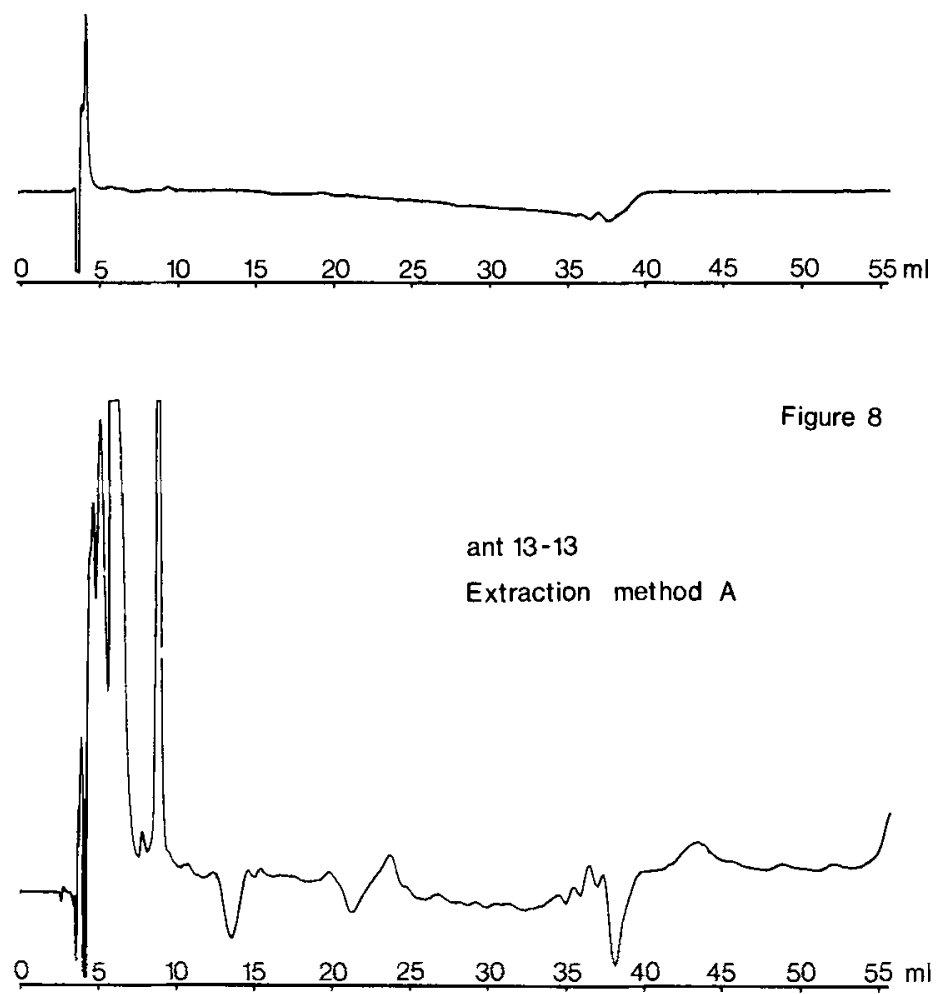

Figures 6-8. HPLC chromatograms of ethyl acetate extract (Fig. 6) and acetone extract (Fig. 8) from the barley mutant ant 13-13. A chromatogram of the solvent is given for comparison (Fig. 7). 
B. Jende-Strid \& B. L. Møller: Proanthocyanidins in barley
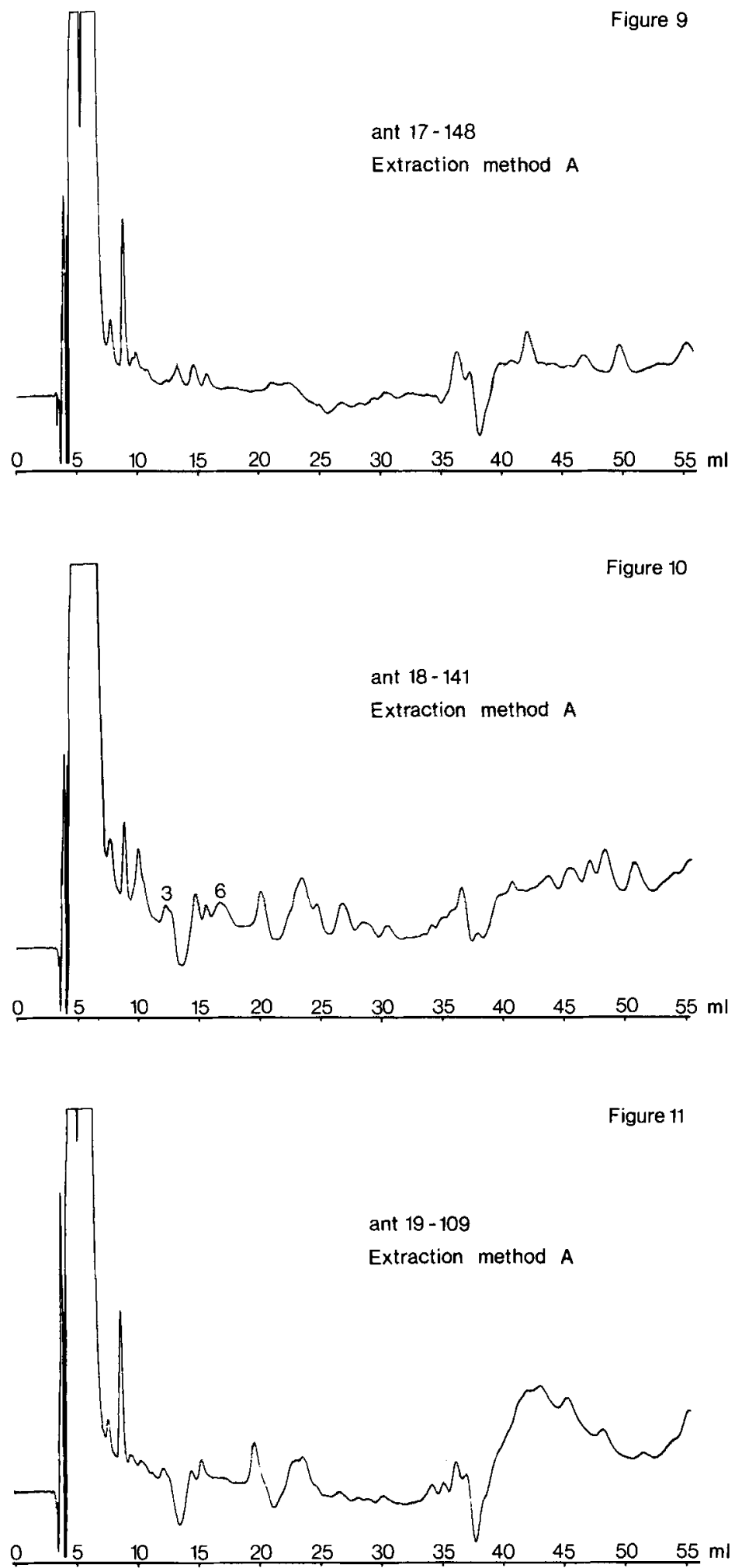

Figures 9-11. HPLC chromatogram of acetone extractable compounds from the mutants ant 17-148, ant 18141 and ant 19-109. 


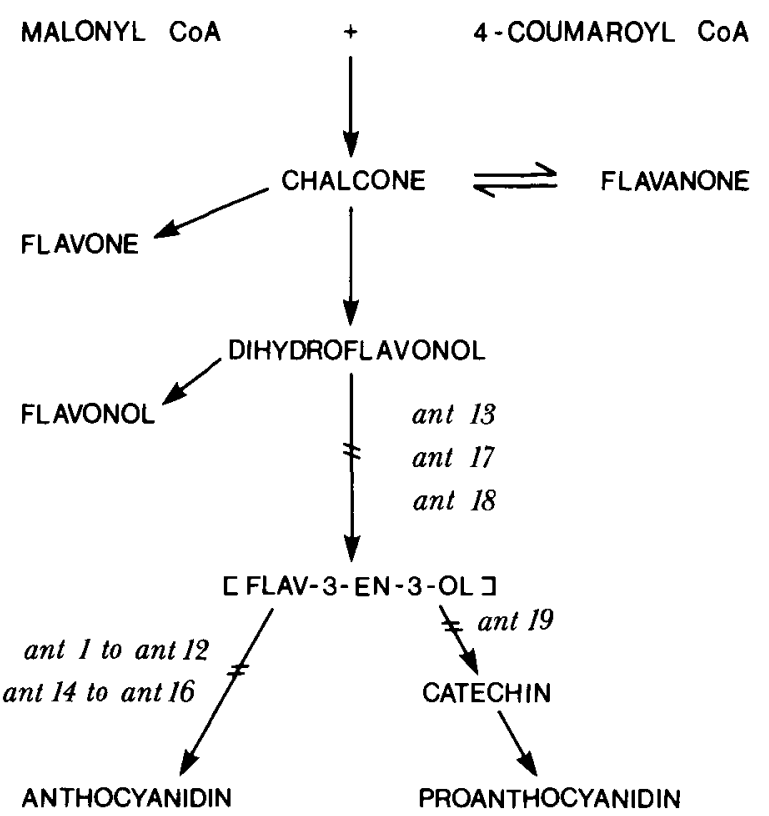

Figure 12. Genetic blocks in the biosynthesis $(6,10$, $11,28)$ of proanthocyanidins caused by mutations in different genes of barley.

malt. OUtrRup $(21,22)$ has isolated and determined the structures of four trimeric barley proanthocyanidins, namely $(+)$-gal-(+)-gal-(+)-cat (trimer 1), $(+)$-gal- $(+)$-cat- $(+)$-cat (trimer 2$),(+)$ cat- $(+)$-gal- $(+)$-cat (trimer 3$)$ and $(+)$-cat- $(+)$ cat- $(+)$-cat (procyanidin $\mathrm{C}-2)$. In addition to the major components catechin, procyanidin B-3, and prodelphinidin B-3 we could confirm the presence of peaks corresponding to the four trimers in wild-type barley. Epicatechin and procyanidin B-6 were not detected. Barley contains at least two additional yet unidentified proanthocyanidins. Considering the results of JoNEs et al. (15) that more than $90 \%$ of the proanthocyanidins in leaves of legumes occur as higher polymers, it will be of interest to determine if also in barley high polymeric forms are dominating.

In beer $80 \%$ of the proanthocyanidins present are derived from barley and the rest from the hops (2). An alternative to stabilization treatments is the use of barley varieties in which the biosynthesis of proanthocyanidins is genetically blocked. Test brews from such barley mutants have resulted in beer with excellent haze stability and taste (29). This is in contrast to the beer obtained after complete removal of all phenolic compounds by extensive filtration through polyvinylpolypyrrolidone which results in a beer with an unacceptable loss of astringency and colour. The HPLC analyses of two mutants in the ant 13 gene document the total loss of proanthocyanidins in these mutants. The majority of proanthocyanidins is also missing in the mutants obtained in three other genes.

Diallelic tests of proanthocyanidin deficient mutants have thus revealed that at least four different genes are involved in blocking the biosynthesis of proanthocyanidins. Mutants in the genes ant 13, ant 17, and ant 18 lack anthocyanin in their green organs and catechin and proanthocyanidins in the aleuron layer of the grain. All three genes block steps prior to the flav-3-en-3-ol intermediate in the pathway of flavonoid biosynthesis (Figure 12). However, the HPLC patterns of acetone extracts obtained from mutants in these three gene loci show small gene specific differences. Mutants in the gene ant-13 (Fig. 8) contain one major unidentified peak (not proanthocyanidin) whereas the chromatograms of mutants in the genes ant 17 (Fig. 9) and ant 18 (Fig. 10) reveal specific complex patterns of unidentified small peaks some of which may represent precursor flavonoids. TLC analysis of unripe grains of ant 13-13 has shown that flavones and other precursor flavonoids are present in ant 13-13 (30). The genes ant 13, ant 17, and ant 18 are probably controlling different steps between the chaicone and the flav-3-en-3-ol intermediate in the flavonoid pathway. The gene ant-19 blocks a step between the flav-3-en-3-ol intermediate and catechin in the flavonoid pathway, i.e. after the branch point leading to anthocyanines. This is evident from the fact that ant 19-109 has a normal content of anthocyanin in the green parts of the plant but no catechin or proanthocyanidin in the grains. Diallelic testing of mutants which are deficient in anthocyanin but have a normal content of catechin and proanthocyanidins in the grains reveal that at least 15 different genes are involved in anthocyanin biosynthesis (12). The proanthocyanidin profiles of such mutants correspond to those of the wild-type. The anthocyanin free barley varieties which today are commercially grown, all belong to this category. 
The present investigation has shown that quantitative and reliable compositional analyses of proanthocyanidins can be carried out routinely by HPLC. This is essential for control measures in the use of proanthocyanidin free barley varieties in malting and brewing. This analytical technique may also aid in identifying mutants containing only a single or a few proanthocyanidin components, which would allow the evaluation of the efficiency of individual components in beer haze formation.

\section{ACKNOWLEDGEMENTS}

Drs. E. Haslam, L. Porter and cand. polyt. H. OutTrup are thanked for providing the proanthocyanidin standards used in this work. Cand. polyt. H. Outrrup is also thanked for helpful discussions and professor D. vON WETTSTEIN for a helpful review of the manuscript.

\section{REFERENCES}

1. Bate-Smith, E. C.: Adstringent tannins of Viburnum and Hydrangea species. Phytochemistry $17,267-270$ (1978)

2. Erdal, K., B. Ahrenst-Larsen \& B. JendeSTRID: Use of proanthocyanidin-free barley in beer brewing. In: Cereals for Food and Beverages. G. E. Inglett \& L. Munck, Eds. Academic Press Inc., pp. 365-379 (1980)

3. Foo, L. Y. \& L. J. Porter: Definition of structural units. J. Chem. Soc. Perkin I, 11861190 (1978)

4. Foo, L. Y. \& L. J. Porter: The phytochemistry of proanthocyanidin polymers. Phytochemistry 19, 1747-1754 (1980)

5. Gramshaw, J. W.: Beer polyphenols and the chemical basis of haze formation I. Phenolic constituents of beer and its raw materials. Tech. Q. Master Brew. Ass. Am. 6, 239-251 (1969)

6. Grisebach, H.: Recent investigations on the biosynthesis of flavonoids. In: Recent Advances in Phytochemistry, Vol. 1. T. J. MABRY, R. E. Alston \& V. C. Runckles, Eds. Appleton Century - Crofts, New York, pp. 379-406 (1968)

7. Gupta, R. K. \& E. Haslam: Plant proanthocyanidins. Part 5. Sorghum polyphenols. J. Chem. Soc. Perkin I, 892-896 (1978)

8. Hardin, J. M. \& C. A. Stutte: Analyses of phenolic and flavonoid compounds by highpressure liquid chromatography. Anal. Biochem. $102,171-175$ (1980)
9. Harris, G. \& R. W. Ricketts: Beer haze - the problem and a solution. Eu. Brew. Con. Proc. Cong., Rome 1959, 290-302 (1959)

10. Haslam, E.: Natural proanthocyanidins. In: The Flavonoids. J. B. HaRborne, T. J. Mabry \& H. MaBRY, Eds. Chapman and Hall, London, pp. 506-559 (1975)

11. Jacques, D., C. T. Opie, L. J. Porter \& E. Haslam: Plant proanthocyanidins. Part 4. Biosynthesis of procyanidins and observations on the metabolism of cyanidin in plants. J. Chem. Soc. Perkin I, 1637-1643 (1977)

12. JENDE-STRID, B.: Diallelic tests of anthocyanindeficient mutants. Barley Genetics Newsletter 8 , 57-58 (1978)

13. JENDE-STRID, B.: Mutations affecting flavonoid synthesis in barley. Carlsberg Res. Commun. 43, 265-273 (1978)

14. Jerumanis, J.: Separation et identification de flavonoides par chromatographie liquide a haute performance (HPLC). Eu. Brew. Con. Proc. Cong., Berlin 1979, 309-319 (1979)

15. Jones, W. T., R. B. Broadhurst \& J. W. LYTTLETON: The condensed tannins of pasture legume species. Phytochemistry 15, 1407-1409 (1976)

16. LaWs, D. R. J., J. D. MCGuinesS \& N. A. Bath: The use of ${ }^{14} \mathrm{C}$-labelled dimeric catechin. J. Am. Soc. Brew. Chem. 34, 170-173 (1976)

17. LEA, G. H. L.: High performance liquid chromatography of cider procyanidins. J. Sci. Food Agric. 30, 833-838 (1979)

18. MCFarlane, W. D.: Determination of anthocyanogens. J. Inst. Brew. 67, 502-506 (1961)

19. McFarlane, W. D., P. F. Sword \& G. Blinoff: Catechin-Tannins in brewing. Eu. Brew. Con. Proc. Cong., Brussels 1963, 174-181 (1963)

20. McMurrough, I.: Chromatographic procedures for measuring polyphenol haze precursors. Eu. Brew. Con. Proc. Cong., Berlin 1979, 321-334 (1979)

21. Outtrup, H. \& K. Schaumburg: Structural elucidation of some proanthocyanidins in barley by ${ }^{1} \mathrm{H} 270 \mathrm{MHz}$ NMR spectroscopy. Carlsberg Res. Commun. 46, 43-52 (1981)

22. Outtrup, H.: Structure of prodelphinidins in barley. Eu. Brew. Con. Cong., Copenhagen (in press) (1981)

23. Price, L. M. \& L. G. Butler: Tannins and nutrition. Bulletin No. 272. Agricultural Experiment Station, Purdue University, West Lafayette, Indiana (1980)

24. Strack, D., K. Fuisting \& G. Popovici: Highperformance liquid chromatographic analysis of oat (Avena sativa) flavone derivatives. J. Chromatog. 176, 270-273 (1979) 
25. Strack, D., N. Akavia \& H. Reznik: High performance liquid chromatographic identification of anthocyanins. Z. Naturforsch. 35c. 533$538(1980)$

26. Thompson, R. S., D. Jacques, E. Haslam \& R. J. N. Tanner: Plant proanthocyanidins. Part I. Introduction: the isolation, structure, and distribution in nature of plant procyanidins. J. Chem. Soc. Perkin I, 1387-1399 (1972)

27. VanCRaenenbroeck, R., M. Kara-Zaitri \& A. Devreux: Influence de la teneur en proanthocyanidines dimers et en catechines de la biere sur la stabilite colloidale. Eu. Brew. Con. Proc. Cong., Berlin 1979, 293-308 (1979)

28. Weinges, K., W. Bähr, W. Ebert, K. Göritz \& H.-D. Marx: Die dimeren Proanthocyanidine. In Fortschritte der Chemie organischer Naturstoffe 27, 234-245 (1969), Springer Verlag, Vienna.
29. Wettstein, D. von, B. Jende-Strid, B. AhRenst-Larsen \& J. A. Sørensen: Biochemical mutant in barley renders chemical stabilization of beer superfluous. Carlsberg Res. Commun. 42, 341-351 (1977)

30. Wettstein, D. von, B. Jende-Strid, B. Ahrenst-Larsen \& K. Erdal: Proanthocyanidinfree barley prevents the formation of beer haze. Tech. Q. Master Brew. Ass. Am. 17, 16-23 (1980)

31. Williams, M., G. Hrazdina, M. M. Wilkinson, J. G. SWeeny \& G. A. IAcobucci: High-pressure liquid chromatographic separation of 3-glucosides, 3,5-diglucosides, 3-(6-0-p-coumaryl) glucosides and 3-(6-o-p-comarylglucoside)-5-glucosides of anthocyanidins. J. Chromatog. 155, 389398 (1978) 